\title{
Cardioprotective Effects of Sarcolemmal and Mitochondrial K-ATP Channel Openers in an Experimental Model of Autoimmune Myocarditis
}

\author{
Role of the Reduction in Calcium Overload During Acute Heart Failure
}

\author{
Shinichi Niwano, ${ }^{1}$ MD, Shoji Hirasawa, ${ }^{1}$ MD, Hiroe Niwano, ${ }^{1}$ MD, Sae Sasaki, ${ }^{1}$ MD, \\ Ray Masuda, ${ }^{3} \mathrm{MD}$, Kiyotaka SATo, ${ }^{2} \mathrm{MD}$, Takashi Masuda, ${ }^{1} \mathrm{MD}$, and Tohru Izumi, ${ }^{1} \mathrm{MD}$
}

\begin{abstract}
SUMMARY
It has been reported that K-ATP channel openers have a cardioprotective effect in acute ischemia as a pharmacological preconditioning effect. In the present study, the chronic effects of clinical K-ATP channel openers, ie, nicorandil (Nic) and mexiletine (Mex), on cardiac function were evaluated in a rat model of experimental autoimmune myocarditis (EAM). Nicorandil (3 or $10 \mathrm{mg} / \mathrm{kg} / \mathrm{day}$ ) or Mex (10 or $25 \mathrm{mg} / \mathrm{kg} /$ day) was administered to the EAM rats, and the effects were compared with those in untreated EAM rats (control EAM) and sham rats without EAM on day 21 (acute phase) or day 60 (chronic phase). In the acute phase, the control EAM rats exhibited a reduced left ventricular ejection fraction (LVEF) and prolonged monophasic action potential duration (MAPD). Neither drug had an affect on the LVEF or degree of myocarditis, but Mex $25 \mathrm{mg}$ suppressed the MAPD prolongation. In the chronic phase, EAM+Nic and EAM+Mex 25 mg exhibited a higher LVEF than the control EAM. Although the control EAM exhibited sustained MAPD prolongation, the other groups showed recovery of the MAPD in the chronic phase. The mitochondorial redox state was lower in the control EAM than in the sham, and EAM+Nic exhibited a similar level of the redox state as the sham in the chronic phase. Nicorandil exhibited a cardioprotective effect through the protection of mitochondrial function. Mexiletine exhibited a cardioprotective effect possibly through a reduction in the calcium overload by shortening the MAPD in the acute phase. (Int Heart J 2012; 53: 139-145)
\end{abstract}

Key words: K-ATP channel, Myocardial protection, Nicorandil, Mexiletine, Myocarditis

$\mathrm{T}$ he K-ATP channel exists in the cell membranes of many organs and has been known to play various roles, such as triggering insulin secretion in the pancreas, or suppressing lymphocytes during inflammatory conditions. ${ }^{1,2}$ In an ischemic myocardium, the sarcolemmal K-ATP channels open, corresponding to a reduction in the intracellular ATP, which prevents myocytes from an intracellular calcium overload by shortening the action potential duration. This response is considered to be an auto-cardioprotective effect during ischemic conditions, which is known to be enhanced by sarcolemmal KATP channel openers. ${ }^{3-7)}$ However, K-ATP channels are found not only in the sarcolemma of myocytes, but also in the mitochondrial membrane, and openers of these mitochondrial KATP channels, such as nicorandil, have been proven to play an important role in its cardioprotective effect by protecting mitochondrial function, especially in short-term acute ischemia. ${ }^{8-10)}$

It has been reported that an increase in ventricular wall stress causes prolongation of the action potential duration, particularly in damaged myocardium with heart failure. ${ }^{11)}$ This prolongation of the action potential duration may increase transient calcium and may exaggerate intracellular calcium overload, resulting in the deterioration of myocardial dysfunction. ${ }^{12)}$ We documented that this electrophysiological change can also be observed in acute inflammatory conditions in an experimental autoimmune myocarditis (EAM) model in rats, ${ }^{13,14)}$ which is a model showing acute myocarditis in the acute phase followed by dilated cardiomyopathy in the chronic phase. In the acute phase of this model, there would be a worsening cycle between the intracellular calcium overload and prolonged action potential duration as a result of the myocardial injury, which may also adversely affect mitochondrial function. Based on these findings, we speculate that shortening of the action potential duration in this acute phase protects the myocardium by decreasing the intracellular calcium overload, and the protection of mitochondrial function prevents myocardial injury, based on similar mechanisms seen in ischemic conditions. In the present study, we evaluated the effects of two clinically available KATP channel openers, nicorandil (Nic: mainly a mitochondrial

From the Departments of ${ }^{1}$ Cardio-Angiology and ${ }^{2}$ Neurology, Kitasato University School of Medicine, Kanagawa, and ${ }^{3}$ Health Control Center, Chuo University, Tokyo, Japan.

This study was supported by a grant for scientific research from the Ministry of Education Science and Culture of Japan (No. 11838015), and also by a grant to the Research Committee for Epidemiology and Etiology of Idiopathic Cardiomyopathy from the Ministry of Health and Welfare of Japan.

Address for correspondence: Shinichi Niwano, MD, Department of Cardio-Angiology, Kitasato University School of Medicine, 1-15-1 Kitasato, Minami-ku, Sagamihara, Kanagawa 252-0374, Japan.

Received for publication November 29, 2011.

Accepted December 15, 2011. 
K-ATP channel opener) and mexiletine (Mex: a sarcolemmal K-ATP channel opener with a sodium channel blocking effect), on ventricular dysfunction in EAM.

\section{Methods}

Induction of autoimmune myocarditis: EAM was induced in 6-week-old male Lewis rats by immunization with purified porcine cardiac myosin as previously described $(n=110) .^{13,14)}$ The sham rats received injections of the same amount of saline $(0.25 \mathrm{~mL})$ in the same manner $(n=20)$. EAM was induced in all 110 rats immunized, however, 7 rats died during the acute phase so 103 of 110 were used in the experiments. The present study was performed in accordance with the Guide for the Care and Use of Laboratory Animals published by the US National Institutes of Health (NIH Publication No. 85-23, revised 1996) and Ethics Committee of the Kitasato University School of Medicine.

Grouping and administration of drugs: The rats were divided into 6 groups in accordance with the induction of EAM and drug administration as follows; 1) control EAM: rats with induction of EAM but without any additional drug administration $(n=28), 2)$ EAM+Nic $3 \mathrm{mg}$ : rats with EAM and treatment with Nic $3 \mathrm{mg} / \mathrm{kg} /$ day $(n=25), 3)$ EAM+Nic $10 \mathrm{mg}$ : rats with EAM and treatment with Nic $10 \mathrm{mg} / \mathrm{kg} /$ day $(n=24), 4)$ EAM+Mex $10 \mathrm{mg}$ : rats with EAM and treatment with Mex 10 $\mathrm{mg} / \mathrm{kg} /$ day $(n=14), 5) \mathrm{EAM}+\mathrm{Mex} 25 \mathrm{mg}$ : rats with EAM and treatment with Mex $25 \mathrm{mg} / \mathrm{kg} /$ day $(n=12)$, and 6) sham: rats without induction of EAM and no additional drug administration $(n=20)$. A study drug was orally administered by adding it to the drinking water after the immunization to induce EAM. The dosage of the drug was calculated by monitoring the water consumption and body weight. The dosages used were determined based on previous reports, ${ }^{15-17)}$ ie, the lower and higher dosages represented the middle and highest doses of the therapeutic range, respectively.

Noninvasive echocardiography: The left ventricular dimension and function in vivo were assessed using transthoracic echocardiography (ProSound SSD-4000, Aloka, Tokyo) as previously described ${ }^{13)}$ and were performed in randomly selected rats at day 21 or day 60 after the initial immunization as summarized in Table I. The assessment was done noninvasively under light anesthesia with an intraperitoneal injection of 2,2,2-tribromoethanol (80-120 mg/kg). The left ventricular end-systolic and end-diastolic dimensions were measured from the M-mode echocardiogram to calculate the left ventricular ejection fraction (LVEF) and fraction shortening (LV\%FS).

Open chest electrophysiological study: After the echocardiographical evaluation, an open chest electrophysiological study

Table I. Histological and Physiological Parameters

\begin{tabular}{|c|c|c|c|c|c|c|}
\hline Day 21 & $\begin{array}{l}\text { Sham } \\
(n=5)\end{array}$ & $\begin{array}{l}\text { Control EAM } \\
\quad(n=9)\end{array}$ & $\begin{array}{c}\text { EAM+Nic } 3 \mathrm{mg} \\
(n=7)\end{array}$ & $\begin{array}{c}\mathrm{EAM}+\mathrm{Nic} 10 \mathrm{mg} \\
(n=7)\end{array}$ & $\begin{array}{c}\text { EAM+Mex } 10 \mathrm{mg} \\
(n=7)\end{array}$ & $\begin{array}{c}\text { EAM+Mex } 25 \mathrm{mg} \\
(n=6)\end{array}$ \\
\hline $\mathrm{Hw} / \mathrm{Bw}(\mathrm{g} / \mathrm{kg})$ & $3.5 \pm 0.3$ & $8.0 \pm 0.6^{*}$ & $7.9 \pm 0.7^{*}$ & $7.8 \pm 0.8^{*}$ & $7.7 \pm 1.0^{*}$ & $8.4 \pm 1.1^{*}$ \\
\hline Area of cellular infiltration $(\%)$ & $0.0 \pm 0.0$ & $37.6 \pm 6.6^{*}$ & $35.2 \pm 7.2^{*}$ & $31.6 \pm 5.6^{*}$ & $35.4 \pm 7.2^{*}$ & $36.1 \pm 6.9^{*}$ \\
\hline Area of fibrosis (\%) & - & - & - & - & - & - \\
\hline Heart rate $(\mathrm{bpm})$ & $376 \pm 36$ & $382 \pm 40$ & $376 \pm 42$ & $386 \pm 39$ & $377 \pm 42$ & $386 \pm 36$ \\
\hline LVSP (mmHg) & $116 \pm 26$ & $104 \pm 25$ & $108 \pm 32$ & $105 \pm 22$ & $106 \pm 26$ & $99 \pm 32^{*}$ \\
\hline LVEDP (mmHg) & $1.9 \pm 0.8$ & $12.1 \pm 5.2^{*}$ & $11.9 \pm 4.8^{*}$ & $12.4 \pm 4.6^{*}$ & $11.6 \pm 4.3^{*}$ & $13.4 \pm 6.8^{*}$ \\
\hline $\operatorname{LVEF}(\%)$ & $76 \pm 5$ & $40 \pm 6^{*}$ & $42 \pm 5^{*}$ & $42 \pm 8^{*}$ & $41 \pm 5^{*}$ & $36 \pm 8^{*}$ \\
\hline LV\%FS & $45 \pm 4$ & $21 \pm 6^{*}$ & $22 \pm 5^{*}$ & $23 \pm 7^{*}$ & $22 \pm 6^{*}$ & $19 \pm 9^{*}$ \\
\hline $\mathrm{ERP}(\mathrm{BCL}=150 \mathrm{~ms}, \mathrm{~ms})$ & $70 \pm 6$ & $84 \pm 9^{*}$ & $83 \pm 7^{*}$ & $81 \pm 6^{*}$ & $79 \pm 8^{*}$ & $75 \pm 6^{\dagger}$ \\
\hline $\mathrm{ERP}(\mathrm{BCL}=120 \mathrm{~ms}, \mathrm{~ms})$ & $67 \pm 5$ & $81 \pm 7^{*}$ & $79 \pm 6^{*}$ & $80 \pm 9^{*}$ & $75 \pm 6^{*}$ & $72 \pm 8^{\dagger}$ \\
\hline $\mathrm{MAPD}_{20}(\mathrm{BCL}=150 \mathrm{~ms}, \mathrm{~ms})$ & $13 \pm 4$ & $30 \pm 4^{*}$ & $28 \pm 6^{*}$ & $26 \pm 5^{*}$ & $24 \pm 5^{*}$ & $18 \pm 7^{* \dagger}$ \\
\hline $\mathrm{MAPD}_{20}(\mathrm{BCL}=120 \mathrm{~ms}, \mathrm{~ms})$ & $14 \pm 3$ & $31 \pm 6^{*}$ & $26 \pm 7^{*}$ & $24 \pm 5^{*}$ & $22 \pm 4^{*}$ & $17 \pm 7^{\dagger}$ \\
\hline $\operatorname{MAPD}_{90}(B C L=150 \mathrm{~ms}, \mathrm{~ms})$ & $62 \pm 5$ & $108 \pm 8^{*}$ & $102 \pm 8^{*}$ & $104 \pm 8^{*}$ & $95 \pm 12^{*}$ & $78 \pm 9^{* \dagger}$ \\
\hline $\operatorname{MAPD}_{90}(\mathrm{BCL}=120 \mathrm{~ms}, \mathrm{~ms})$ & $60 \pm 6$ & $104 \pm 10^{*}$ & $104 \pm 10^{*}$ & $99 \pm 10^{*}$ & $96 \pm 11^{*}$ & $74 \pm 12^{* \dagger}$ \\
\hline Day 60 & $\begin{array}{l}\text { Sham } \\
(n=5)\end{array}$ & $\begin{array}{l}\text { Control EAM } \\
\quad(n=9)\end{array}$ & $\begin{array}{c}\mathrm{EAM}+\mathrm{Nic} 3 \mathrm{mg} \\
(n=8)\end{array}$ & $\begin{array}{c}\mathrm{EAM}+\mathrm{Nic} 10 \mathrm{mg} \\
(n=7)\end{array}$ & $\begin{array}{c}\text { EAM+Mex } 10 \mathrm{mg} \\
(n=7)\end{array}$ & $\begin{array}{c}\text { EAM+Mex } 25 \mathrm{mg} \\
(n=6)\end{array}$ \\
\hline $\mathrm{Hw} / \mathrm{Bw}(\mathrm{g} / \mathrm{kg})$ & $3.5 \pm 0.5$ & $5.0 \pm 0.6^{*}$ & $4.0 \pm 0.5^{\dagger}$ & $3.7 \pm 0.4^{\dagger}$ & $4.4 \pm 0.7^{*}$ & $3.9 \pm 0.8^{\dagger}$ \\
\hline Area of cellular infiltration $(\%)$ & $0.0 \pm 0.0$ & $2.6 \pm 2.2$ & $1.6 \pm 1.5$ & $1.4 \pm 1.8$ & $2.6 \pm 1.4$ & $1.6 \pm 2.1$ \\
\hline Area of fibrosis $(\%)$ & $0.0 \pm 0.0$ & $29.6 \pm 5.6^{*}$ & $18.6 \pm 4.6^{*}$ & $8.2 \pm 4.3^{\dagger}$ & $16.6 \pm 6.2^{*}$ & $14.4 \pm 6.2^{*}$ \\
\hline Heart rate (bpm) & $381 \pm 46$ & $368 \pm 36$ & $377 \pm 42$ & $379 \pm 40$ & $368 \pm 36$ & $359 \pm 46$ \\
\hline LVSP (mmHg) & $122 \pm 30$ & $106 \pm 28$ & $110 \pm 26$ & $119 \pm 26$ & $116 \pm 21$ & $111 \pm 24$ \\
\hline LVEDP (mmHg) & $2.0 \pm 1.0$ & $13.9 \pm 8.2^{*}$ & $6.4 \pm 3.4^{*}$ & $3.4 \pm 2.9^{\dagger}$ & $8.6 \pm 4.2^{*}$ & $3.9 \pm 3.0^{\dagger}$ \\
\hline $\operatorname{LVEF}(\%)$ & $75 \pm 6$ & $37 \pm 6^{*}$ & $62 \pm 6^{\dagger}$ & $72 \pm 8^{\dagger}$ & $46 \pm 7^{*}$ & $67 \pm 9^{\dagger}$ \\
\hline LV\%FS & $43 \pm 7$ & $20 \pm 5^{*}$ & $41 \pm 3^{\dagger}$ & $44 \pm 6^{\dagger}$ & $26 \pm 7^{*}$ & $40 \pm 6^{\dagger}$ \\
\hline $\mathrm{ERP}(\mathrm{BCL}=150 \mathrm{~ms}, \mathrm{~ms})$ & $71 \pm 6$ & $74 \pm 8$ & $74 \pm 4$ & $70 \pm 5$ & $73 \pm 4$ & $68 \pm 6$ \\
\hline $\mathrm{ERP}(\mathrm{BCL}=120 \mathrm{~ms}, \mathrm{~ms})$ & $67 \pm 5$ & $70 \pm 7$ & $71 \pm 6$ & $68 \pm 7$ & $69 \pm 5$ & $65 \pm 9$ \\
\hline $\operatorname{MAPD}_{20}(\mathrm{BCL}=150 \mathrm{~ms}, \mathrm{~ms})$ & $14 \pm 3$ & $24 \pm 5^{*}$ & $20 \pm 4^{*}$ & $16 \pm 4$ & $19 \pm 3$ & $16 \pm 5$ \\
\hline $\mathrm{MAPD}_{20}(\mathrm{BCL}=120 \mathrm{~ms}, \mathrm{~ms})$ & $15 \pm 3$ & $21 \pm 4^{*}$ & $19 \pm 5$ & $16 \pm 4$ & $17 \pm 5$ & $15 \pm 4$ \\
\hline $\mathrm{MAPD}_{90}(\mathrm{BCL}=150 \mathrm{~ms}, \mathrm{~ms})$ & $65 \pm 6$ & $69 \pm 8$ & $66 \pm 7$ & $66 \pm 9$ & $67 \pm 9$ & $67 \pm 7$ \\
\hline $\mathrm{MAPD}_{90}(\mathrm{BCL}=120 \mathrm{~ms}, \mathrm{~ms})$ & $62 \pm 4$ & $67 \pm 8$ & $65 \pm 6$ & $63 \pm 7$ & $65 \pm 6$ & $63 \pm 9$ \\
\hline
\end{tabular}

${ }^{*} P<0.05$ versus sham, ${ }^{\dagger} P<0.05$ versus Control EAM. Hw/Bw indicates heart and body weight ratio; LVSP, left ventricular systolic pressure; LVEDP, left ventricular end-diastolic pressure; LVEF, leftventricular ejection fraction; LV\%FS, left ventricular percent fraction shortening; ERP, effective refractory period; BCL, basic cycle length, and MAPD, duration of the monophasic action potential. 
was performed to evaluate the electrophysiological parameters on day 21 or day 60 as summarized in Table I. A median sternotomy was carried out under intraperitoneal anesthesia with 2,2,2-tribromoethanol ( $240 \mathrm{mg} / \mathrm{kg})$. To avoid the influence of these macroscopic procedures on mitochondrial function, the rats used in the assessment of mitochondrial function were prepared separately from these rats for the electrophysiological measurements as shown in Table II. For the hemodynamic parameters, the left ventricular systolic pressure (LVSP) and left ventricular end-diastolic pressure (LVEDP) were monitored by a needle tip micromanometer (SPR477, Millar, USA) as previously described. ${ }^{13,14)}$ For the electrophysiological evaluation, a pair of platinum needle electrodes $(\varphi 0.1 \mathrm{~mm})$ was directly inserted into the left ventricular free wall, and used for electrical stimulation and recording. The analogue signals were converted into digital signals at a sampling frequency of $1000 \mathrm{~Hz}$ (Power Lab 8sp, Bio Research, Tokyo) and stored on a computer hard disk. The band pass filter was set at $50-300 \mathrm{~Hz}$ for a standard electrocardiogram recording and at an open- $300 \mathrm{~Hz}$ for recording the monophasic action potential (MAP). To evaluate the ventricular effective refractory period (ERP), a $2 \mathrm{~ms}$ step shortening the coupling interval of the extrastimulus was employed in two basic cycle lengths of 150 and $120 \mathrm{~ms}$. The MAP duration (MAPD) was determined as the interval from the onset of the MAP to $20 \%$ of the repolarization time $\left(\mathrm{MAPD}_{20}\right)$ or to its $\left.90 \%\left(\mathrm{MAPD}_{90}\right)\right)^{13,14)}$

Heart weight and histology: After the electrophysiological measurements, the heart was totally excised and the weight of the whole heart was measured to calculate the ratio of the heart to body weight $(\mathrm{Hw} / \mathrm{Bw})$. The heart was fixed with $10 \%$ formalin and embedded in paraffin. After the heart was sliced transversely, the tissue was stained with hematoxylin-eosin or Azan-Mallory for histological evaluation. The area infiltrated by inflammatory cells and/or fibrosis was evaluated using Mac SCOPE software (Mitani Co., Japan) and was expressed as the percentage of the whole ventricular area. ${ }^{4,13)}$

Evaluation of mitochondrial function: In randomly selected rats, the contents of the metabolites in the ventricular myocardium were enzymatically measured to evaluate the mitochondrial function on day 21 or day 60 as summarized in Table II. Since Mex, a sarcolemmal K-ATP channel opener, rarely affects mitochondrial function, it was not assessed in the rats treated with Mex. The ventricular muscle was freeze clamped in liquid nitrogen immediately after the median sternotomy and stored at $-80^{\circ} \mathrm{C}$ until measurement of the myocardial metabolites. The cardiac muscle was extracted with $3.6 \%$ perchloric acid and neutralized, followed by enzymatic measurement of adenosine-triphosphate (ATP), phosphocreatinine (P-Cr), creatinine $(\mathrm{Cr})$, pyruvate (Pyr), lactate (Lac), dihydroxyacetone phosphate (DHAP), 3-phosphoglycerate (3-PG), $\alpha$-ketoglutarate $(\alpha-\mathrm{KG})$, glutamate, and ammonia $\left(\mathrm{NH}_{4}\right)$ as previously described. ${ }^{18,19)}$ The myocardial contents of ADP and Pi, the [ATP]/ [ADP] ratio, cytosolic phosphorylation potential (PP), ie, $[\mathrm{ATP}] /[\mathrm{ADP}] /[\mathrm{Pi}]$, mitochondrial redox state $\left(\mathrm{mit}\left[\mathrm{NAD}^{+}\right] /\right.$

Table II. Metabolites in the Myocardium

\begin{tabular}{|c|c|c|c|c|}
\hline Day 21 & $\begin{array}{l}\text { Sham } \\
(n=5)\end{array}$ & $\begin{array}{l}\text { Control EAM } \\
\quad(n=5)\end{array}$ & $\begin{array}{c}\mathrm{EAM}+\mathrm{Nic} 3 \mathrm{mg} \\
(n=5)\end{array}$ & $\begin{array}{c}\mathrm{EAM}+\mathrm{Nic} 10 \mathrm{mg} \\
(n=5)\end{array}$ \\
\hline ATP ( $\mu \mathrm{mol} / \mathrm{g}$ wet tissue $)$ & $2.14 \pm 0.08$ & $1.65 \pm 0.05^{* *}$ & $1.65 \pm 0.09^{*}$ & $1.68 \pm 0.05^{*}$ \\
\hline $\mathrm{P}-\mathrm{Cr}(\mu \mathrm{mol} / \mathrm{g}$ wet tissue $)$ & $6.31 \pm 0.38$ & $3.62 \pm 0.36^{*}$ & $3.65 \pm 0.33^{*}$ & $3.88 \pm 0.28^{*}$ \\
\hline Pyruvate ( $\mu \mathrm{mol} / \mathrm{g}$ wet tissue) & $0.051 \pm 0.003$ & $0.035 \pm 0.003^{*}$ & $0.032 \pm 0.002^{*}$ & $0.036 \pm 0.003$ \\
\hline Lactate ( $\mu \mathrm{mol} / \mathrm{g}$ wet tissue) & $0.54 \pm 0.02$ & $0.65 \pm 0.03$ & $0.62 \pm 0.03$ & $0.65 \pm 0.03$ \\
\hline DHAP ( $\mu \mathrm{mol} / \mathrm{g}$ wet tissue) & $0.040 \pm 0.004$ & $0.020 \pm 0.002^{*}$ & $0.021 \pm 0.003^{*}$ & $0.019 \pm 0.003^{*}$ \\
\hline 3PG $(\mu \mathrm{mol} / \mathrm{g}$ wet tissue $)$ & $0.019 \pm 0.001$ & $0.014 \pm 0.002$ & $0.013 \pm 0.002$ & $0.013 \pm 0.002$ \\
\hline Glutamate ( $\mu \mathrm{mol} / \mathrm{g}$ wet tissue) & $2.61 \pm 0.09$ & $2.07 \pm 0.09^{*}$ & $2.23 \pm 0.09$ & $2.36 \pm 0.09$ \\
\hline$\alpha-\mathrm{KG}$ (mmol/g wet tissue) & $0.037 \pm 0.003$ & $0.057 \pm 0.004$ & $0.054 \pm 0.004$ & $0.051 \pm 0.002$ \\
\hline $\mathrm{NH}_{4}(\mathrm{mmol} / \mathrm{g}$ wet tissue $)$ & $0.97 \pm 0.005$ & $1.53 \pm 0.08^{*}$ & $1.51 \pm 0.009^{*}$ & $1.33 \pm 0.06^{*}$ \\
\hline$\Delta \mathrm{G}$ ATP hy $(\mathrm{kcal} / \mathrm{mol})$ & $15.7 \pm 0.1$ & $15.1 \pm 0.1^{*}$ & $15.2 \pm 0.1^{*}$ & $15.1 \pm 0.1^{*}$ \\
\hline mito-[NAD $\left.{ }^{+}\right] /[\mathrm{NADH}]$ & $8.14 \pm 0.77$ & $24.79 \pm 1.44^{* *}$ & $21.39 \pm 1.36^{* *}$ & $17.51 \pm 1.73^{*}$ \\
\hline Eh-mito-[NAD $\left.{ }^{+}\right] /[\mathrm{NADH}](\mathrm{mV})$ & $292.3 \pm 1.3$ & $277.3 \pm 0.9^{* *}$ & $279.7 \pm 1.0^{* *}$ & $282.1 \pm 1.4^{*}$ \\
\hline Day 60 & $\begin{array}{l}\text { Sham } \\
(n=5)\end{array}$ & $\begin{array}{l}\text { Control EAM } \\
(n=5)\end{array}$ & $\begin{array}{c}\mathrm{EAM}+\mathrm{Nic} 3 \mathrm{mg} \\
(n=5)\end{array}$ & $\begin{array}{c}\mathrm{EAM}+\mathrm{Nic} 10 \mathrm{mg} \\
(n=5)\end{array}$ \\
\hline ATP ( $\mu \mathrm{mol} / \mathrm{g}$ wet tissue $)$ & $2.24 \pm 0.07$ & $1.76 \pm 0.06^{*}$ & $2.42 \pm 0.06^{\dagger \dagger}$ & $2.26 \pm 0.07^{\dagger}$ \\
\hline $\mathrm{P}-\mathrm{Cr}(\mu \mathrm{mol} / \mathrm{g}$ wet tissue $)$ & $6.33 \pm 0.33$ & $4.30 \pm 0.35^{*}$ & $6.12 \pm 0.32^{\dagger}$ & $6.20 \pm 0.33^{\dagger}$ \\
\hline Pyruvate $(\mu \mathrm{mol} / \mathrm{g}$ wet tissue $)$ & $0.050 \pm 0.004$ & $0.041 \pm 0.004$ & $0.050 \pm 0.003$ & $0.052 \pm 0.004$ \\
\hline Lactate ( $\mu \mathrm{mol} / \mathrm{g}$ wet tissue) & $0.52 \pm 0.03$ & $0.59 \pm 0.04$ & $0.55 \pm 0.03$ & $0.52 \pm 0.04$ \\
\hline DHAP ( $\mu \mathrm{mol} / \mathrm{g}$ wet tissue) & $0.045 \pm 0.005$ & $0.030 \pm 0.003$ & $0.044 \pm 0.003$ & $0.043 \pm 0.002$ \\
\hline 3PG ( $\mu \mathrm{mol} / \mathrm{g}$ wet tissue) & $0.025 \pm 0.005$ & $0.019 \pm 0.001$ & $0.027 \pm 0.002$ & $0.024 \pm 0.002$ \\
\hline Glutamate $(\mu \mathrm{mol} / \mathrm{g}$ wet tissue $)$ & $2.56 \pm 0.10$ & $2.05 \pm 0.11$ & $2.36 \pm 0.10$ & $2.53 \pm 0.12$ \\
\hline$\alpha-\mathrm{KG}$ (mmol/g wet tissue) & $0.039 \pm 0.003$ & $0.043 \pm 0.002$ & $0.043 \pm 0.003$ & $0.038 \pm 0.003$ \\
\hline $\mathrm{NH}_{4}(\mathrm{mmol} / \mathrm{g}$ wet tissue $)$ & $0.946 \pm 0.051$ & $1.212 \pm 0.059$ & $1.216 \pm 0.055$ & $0.979 \pm 0.053$ \\
\hline DG ATP hy (kcal/mol) & $15.7 \pm 0.1$ & $15.3 \pm 0.1^{*}$ & $15.5 \pm 0.1$ & $15.6 \pm 0.1^{\dagger}$ \\
\hline mito-[NAD $]$ ]/[NADH] & $8.36 \pm 0.22$ & $15.30 \pm 1.29^{*}$ & $13.58 \pm 1.30$ & $8.92 \pm 0.77^{\dagger}$ \\
\hline Eh-mito-[NAD $\left.{ }^{+}\right] /[\mathrm{NADH}](\mathrm{mV})$ & $291.6 \pm 0.4$ & $284.0 \pm 1.1^{*}$ & $285.7 \pm 1.2$ & $291.5 \pm 1.4^{\dagger}$ \\
\hline
\end{tabular}

${ }^{*} P<0.05$ and ${ }^{* *} P<0.01$ versus sham,${ }^{\dagger} P<0.05$ and ${ }^{\dagger} P<0.01$ versus Control EAM. ATP indicates adenosine-triphosphate; $\mathrm{P}-\mathrm{Cr}$, phosphocreatinine; DHAP, dihydroxyacetone phosphate; 3PG, 3-phosphoglycerate; $\alpha$-KG, $\alpha$-ketoglutamate; $\mathrm{NH}_{4}$, ammonia; $\Delta \mathrm{G}$ ATP hy, G-ATP hydrolysis energy; mito [NAD+]/[NADH], mitochondrial redox state; and Eh-mito-[NAD+]/[NADH], mitochondrial redox state of [NAD+]/[NADH]. 
[NADH]), mitochondrial redox potential of $\left[\mathrm{NAD}^{+}\right] /[\mathrm{NADH}]$ $\left(\mathrm{Eh}_{\mathrm{NAD}+\mathrm{NADH}}\right)$, and $\Delta \mathrm{G}_{\mathrm{ATP} \text { hydrolysis }}$ energy $\left(\Delta \mathrm{G}_{\mathrm{ATP} \text { hydr }}\right)$, which is the change in the Gibbs free energy of the ATP hydrolysis in the cytosol of the cells, were calculated as previously reported. ${ }^{18,19)}$ The myocardial metabolites are expressed as the content per $1 \mathrm{~g}$ wet weight of myocardium.

Calculation formulae

Cytosolic $[A D P]=[A T P] \times[C r] /[\mathrm{P}-\mathrm{Cr}] \times 1.27 \times 10^{-2} \mathrm{M}$

Cytosolic $[A T P] /[A D P]=[\mathrm{P}-\mathrm{Cr}] /[\mathrm{Cr}] \times 78.9$

Cytosolic $[\mathrm{Pi}]=[3-\mathrm{PG}] \times[\mathrm{Lac}] \times[\mathrm{P}-\mathrm{Cr}] /[\mathrm{DHAP}] /[\mathrm{Pyr}] /$

$[\mathrm{Cr}] \times 1.45 \times 10^{-4} \mathrm{M}$

Mitochondrial $\left[N A D^{+}\right] /[N A D H]=[\alpha-K G] /\left[\mathrm{NH}_{4}^{+}\right] \times\left[\mathrm{H}^{+}\right] /$

[Glutamate] / $K_{G L D H}$

$E h_{N A D+/ N A D H}=E_{N A D+/ N A D H}^{o}+R T / n F \times \ln \left(\left[N A D^{+}\right] /[N A D H]\right) \times$ $10^{-7} /\left[\mathrm{H}^{+}\right] \mathrm{mV}$

Phosphorylation Potential $=[$ DHAP $] /[$ Pyr $] /[3-P G] /[$ Lac $]$ $\times 5.45 \times 10^{5} M^{1}$

$\Delta G_{A T P h y d r}=\Delta G^{o}+R T \times \ln ([A D P] \times[P i] /[A T P]) \mathrm{kcal} / \mathrm{mol}$

(Pi: inorganic phosphate, NAD: nicotinamide adenine dinucleotide, $\mathrm{NAD}^{+}$: the oxidized form of NAD, NADH: the reduced form of $\mathrm{NAD}, \mathrm{K}$ : the equilibrium constant for the enzyme catalyzed reaction, $\mathrm{K}_{\mathrm{GLDH}}$ : equilibrium constant of the glutamate dehydrogenase reaction)

Statistical analysis: All quantitative data are described as the mean \pm SEM. The basic comparative statistics were performed using an unpaired Student's $t$ test and a $P<0.05$ was considered as statistically significant.

\section{RESUltS}

Heart weight and histology: Figure 1 shows representative examples of the histological findings of the sliced ventricles of the rats in each group on days 21 and 60 . Because the histological findings in EAM can be characterized by infiltration of inflammatory cells in the acute phase (day 21) and tissue fibrosis in the chronic phase (day 60), HE and Azan staining were

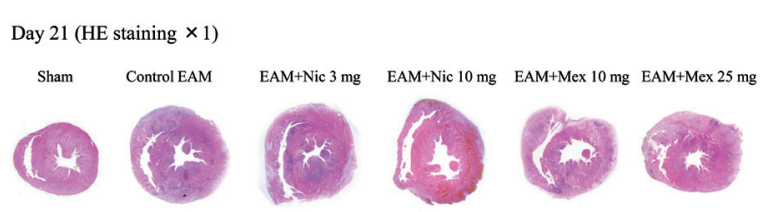

Day 60 (Azan staining $\times 1$ )

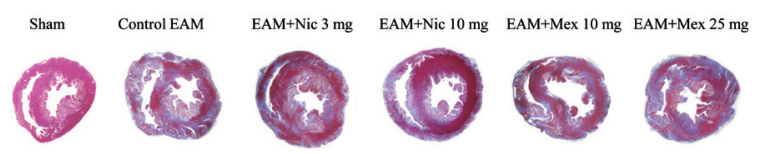

Figure 1. Representative examples of sliced ventricles on days 21 and 60 This figure shows representative examples of the histological findings of the sliced ventricles of rats in each group on days 21 and 60. HE staining was chosen for the presentation of day 21 to exhibit any inflammatory cellular infiltration and Azan staining for day 60 to exhibit any tissue fibrosis. On day 21, the rats with EAM exhibited typical findings of acute myocarditis and were not affected by treatment with Nic or Mex. On day 60, the rats with EAM exhibited tissue fibrosis and were not affected by treatment with Nic or Mex except in the EAM+Nic $10 \mathrm{mg}$ rats. See text for details. HE indicates hematoxylin-eosin; EAM, experimental autoimmune myocarditis; Nic, nicorandil; and Mex, mexiletine. chosen for the presentations of examples, respectively. On day 21, the rats with EAM exhibited a general enlargement due to tissue edema and inflammatory cellular infiltration in comparison with the sham. The treatment with Nic or Mex did not affect the appearance of these findings of acute myocarditis. On day 60 , the rats with EAM now exhibited tissue fibrosis represented by a purple color in comparison with the sham. Similar to day 21, treatment with Nic or Mex did not strongly affect the degree of fibrosis, but the area of the fibrosis in the EAM+Nic $10 \mathrm{mg}$ rats seemed to be somewhat smaller than that in the control EAM rats in this example.

Table I shows the histological and physiological data of the rats on day 21 and day 60. On day 21, the heart and body weight ratio $(\mathrm{Hw} / \mathrm{Bw})$ was higher in the groups with EAM than in the sham and there was no difference among the groups with EAM regardless of the treatment with Nic or Mex. In contrast, on day 60 , the $\mathrm{Hw} / \mathrm{Bw}$ was still higher in the control $\mathrm{EAM}$ and $\mathrm{EAM}+\mathrm{Mex} 10 \mathrm{mg}$ groups than in the sham, but it was significantly smaller in the EAM+Nic $3 \mathrm{mg}$, EAM+Nic $10 \mathrm{mg}$, and EAM+Mex $24 \mathrm{mg}$ groups than in the control EAM group. In the histological findings, the area of the inflammatory cellular infiltration on day 21 was larger in the groups with EAM than in the sham and it was not affected by the treatment with Nic or Mex. On day 60, the cellular infiltration was already negligible in all groups, but the area of the tissue fibrosis was significantly larger in the groups with EAM than in the sham regardless of the treatment with Nic or Mex, with the exception of the group with EAM+Nic $10 \mathrm{mg}$.
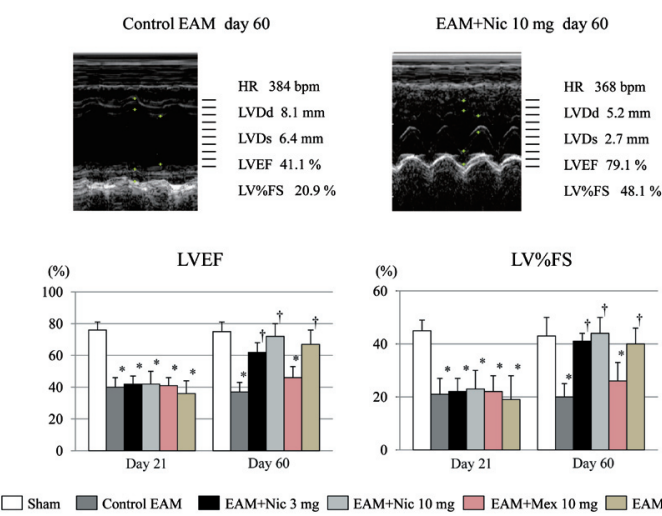

$\square$ Sham $\square$ Control EAM

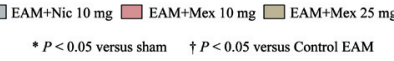

Figure 2. Echocardiographical findings. This figure exhibits the echocardiographical findings of each group on day 21 or day 60 . The upper panels show representative examples of the recordings on day 60 in rats in the control EAM and EAM+Nic $10 \mathrm{mg}$ groups. Although the control EAM rats (left panel) exhibited severe hypokinesia of the ventricular wall motion, the $\mathrm{EAM}+\mathrm{Nic} 10 \mathrm{mg}$ rats exhibited almost normal ventricular wall motion. The lower graphs exhibit the summary data of the LVEF and LV\%FS in each group. On day 21, the groups with EAM exhibited a lower LVEF and LV\%FS than the sham group regardless of the treatment with Nic or Mex. In contrast, on day 60, although the LVEF and LV\%FS were still lower in the control EAM and EAM+Mex $10 \mathrm{mg}$ groups than in the sham group, the EAM+Nic $3 \mathrm{mg}$, EAM+Nic $10 \mathrm{mg}$, and EAM+Mex 25 mg groups exhibited a higher LVEF and LV\%FS than the control EAM group. See text for details. EAM indicates experimental autoimmune myocarditis; Nic, nicorandil; LVEF, left ventricular ejection fraction; LV\%FS, left ventricular percentage of fraction shortening; and Mex, mexiletine. 


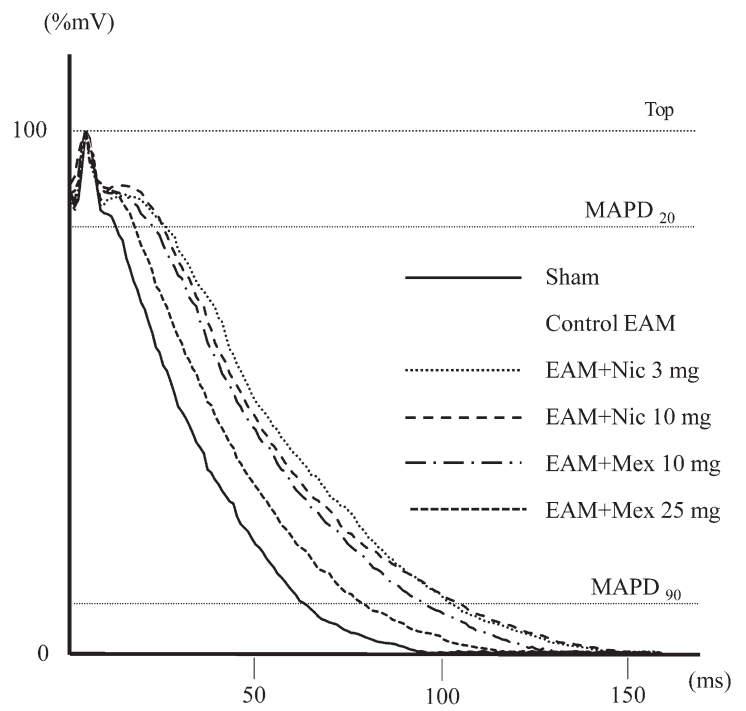

Figure 3. Representative examples of MAP traces on day 21. This figure exhibits representative examples of MAP traces of ventricular muscle in each group on day 21. The control EAM rats exhibited a more prolonged MAPD than the sham rats. The prolonged MAPD was significantly shortened by Mex $25 \mathrm{mg}$. See text for details. MAPD indicates duration of the monophasic action potential; EAM, experimental autoimmune myocarditis; Nic, nicorandil; and Mex, mexiletine.

Hemodynamic and echocardiographical findings: Figure 2 presents the echocardiographical findings of each group on day 21 or day 60 . The upper panels show the representative examples of the recordings on day 60 in the rats in the control EAM and EAM+Nic $10 \mathrm{mg}$ groups, and the lower graphs exhibit the summary data of the LVEF and LV\%FS in each group. The numbers of rats and data from each group are also shown in Table I. On day 21, the groups with EAM exhibited a lower LVEF and LV\%FS than the sham group regardless of the treatment with Nic or Mex and there was no difference among the 5 groups with EAM. In contrast, on day 60, although the LVEF and LV\%FS were still lower in the control EAM and EAM+ Mex $10 \mathrm{mg}$ groups than in the sham group, the groups with $\mathrm{EAM}+\mathrm{Nic} 3 \mathrm{mg}$, EAM+Nic $10 \mathrm{mg}$, and $\mathrm{EAM}+\mathrm{Mex} 25 \mathrm{mg}$ exhibited a higher LVEF and LV\%FS than the control EAM group, and no significant differences were observed in these parameters even in comparisons with the sham group.

Among the hemodynamic parameters, the heart rate did not show any significant difference among any groups on days 21 and 60. The LVSP did not show any difference among the groups either on day 21 or day 60, except for in the EAM+Mex $25 \mathrm{mg}$ group on day 21 in which the value was lower than that in the sham. The LVEDP was higher in the groups with EAM than in the sham on day 21 regardless of the treatment with Nic or Mex. On day 60, the LVEDP was still higher in the control EAM, EAM+Nic $3 \mathrm{mg}$, and EAM+Mex $10 \mathrm{mg}$ groups than in the sham group, but it was lower in the EAM+Nic 10 $\mathrm{mg}$ and EAM+Mex $25 \mathrm{mg}$ groups than in the control EAM (Table II).

Electrophysiological parameters: Figure 3 presents representative examples of MAP traces of the ventricular muscle in each group on day 21 . The MAP trace in the sham rats exhibit-
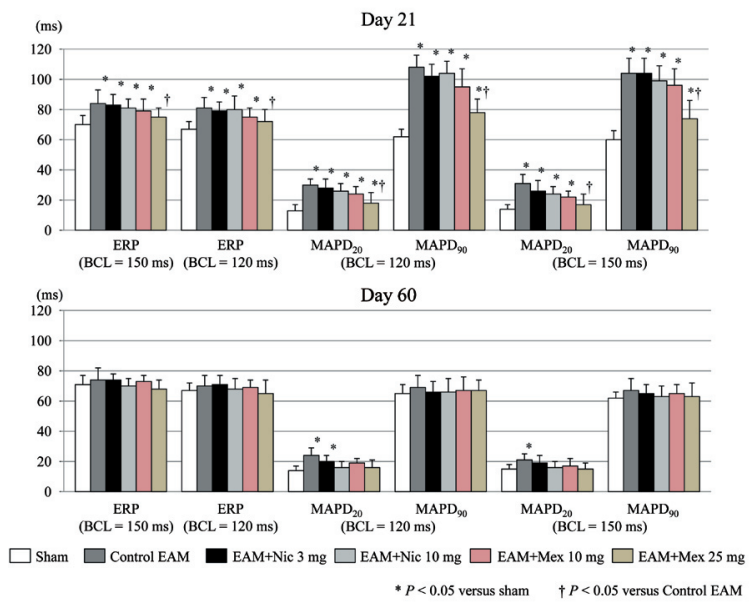

Figure 4. Summary of electrophysiological data. Summary of the ERP and MAPD data in all groups on days 21 and 60. On day 21, the groups with EAM exhibited a prolonged ERP and MAPD in comparison with the sham group, but the Mex treatment partly suppressed this prolongation and the EAM+Mex $25 \mathrm{mg}$ group exhibited a significantly shorter ERP and MAPD than the control EAM group. On day 60, there was no significant difference in the ERP and $\mathrm{MAPD}_{90}$ data among all groups, but the EAM+Nic $3 \mathrm{mg}$ and EAM+Nic $10 \mathrm{mg}$ groups exhibited sustained prolongation of the $\mathrm{MAPD}_{20}$ even in this phase. See text for details. ERP indicates effective refractory period; BCL, basic cycle length; MAPD, duration of the monophasic action potential; Nic, nicorandil; and Mex, mexiletine.

ed a relatively short MAPD, but it was prolonged in the control EAM rats as a result of a "dome" like prolongation in phase 2 of the action potential as previously described. ${ }^{14)}$ We expected that the MAPD would be shortened by the administration of Nic or Mex because of their opening effect on sarcolemmal KATP channels, however, the administration of Nic did not result in any significant shortening of the MAPD even with a higher dose (ie, $10 \mathrm{mg} / \mathrm{kg} /$ day in this study). As a result, the treatment with a higher dose of Mex (ie, $25 \mathrm{mg} / \mathrm{kg} / \mathrm{day}$ in this study) caused a significant shortening of the MAPD in comparison with the control EAM.

Figure 4 and Table I summarize the ERP and MAPD data in all groups on days 21 and 60. On day 21, the groups with EAM exhibited a prolonged ERP and MAPD in comparison with the sham group. However, this prolongation was partly suppressed by Mex treatment and the EAM+Mex $25 \mathrm{mg}$ group exhibited a significantly shorter ERP and MAPD than the control EAM group. On day 60, there was no significant difference in the ERP and $\mathrm{MAPD}_{90}$ data among the groups, but the $\mathrm{EAM}+\mathrm{Nic} 3 \mathrm{mg}$ and $\mathrm{EAM}+\mathrm{Nic} 10 \mathrm{mg}$ groups exhibited a sustained prolongation of the $\mathrm{MAPD}_{20}$ even in this phase.

Mitochondrial function: Table II summarizes the myocardial contents of the metabolites and presents variables in the myocardial mitochondria in the sham, control EAM, EAM+Nic 3 $\mathrm{mg}$, and EAM+Nic $10 \mathrm{mg}$ groups. The number of rats in each group is shown in the table. On day 21, the levels of ATP, P-Cr, $\Delta \mathrm{G}_{\mathrm{ATP} h y d r}$, and $\mathrm{Eh}_{\mathrm{NAD}+\mathrm{NADH}}$ decreased and the mit $\left[\mathrm{NAD}^{+}\right] /$ [NADH] increased in all groups with EAM regardless of the treatment with $\mathrm{Nic}$, in comparison with the sham group. In contrast, on day 60, although the control EAM group exhibited still lower levels of ATP and P-Cr than the sham group, their 
levels in the $\mathrm{EAM}+\mathrm{Nic} 3 \mathrm{mg}$ and $\mathrm{EAM}+\mathrm{Nic} 10 \mathrm{mg}$ groups recovered and became significantly higher than in the control EAM group. $\Delta \mathrm{G}_{\mathrm{ATP} \text { hydr }}$ and $\mathrm{Eh}_{\mathrm{NAD}+\mathrm{NADH}}$ were lowest and the mit $\left[\mathrm{NAD}^{+}\right] /[\mathrm{NADH}]$ was highest in the control EAM group on day 60, while those in the EAM+Nic $3 \mathrm{mg}$ and EAM+Nic 10 $\mathrm{mg}$ groups were at similar levels to the sham group. Furthermore, $\Delta \mathrm{G}_{\mathrm{ATP} \text { hydr }}$ and $\mathrm{Eh}_{\mathrm{NAD+} / \mathrm{NADH}}$ were significantly higher and mit $\left[\mathrm{NAD}^{+}\right] /[\mathrm{NADH}]$ was significantly lower in the EAM+Nic $10 \mathrm{mg}$ group than in the control EAM group.

\section{DiscusSION}

This study evaluated the effects of two K-ATP channel openers, nicorandil (Nic) and mexiletine (Mex), on left ventricular dysfunction in rats with EAM. To the best of our knowledge, this is the first systematic study evaluating the effect of the chronic use of K-ATP channel openers on myocarditis and long-lasting left ventricular dysfunction. Several interesting findings were observed. First, the treatment with Nic or Mex did not affect the degree of acute myocarditis during its acute phase (day 21), but the tissue fibrosis in the chronic phase (day 60) was suppressed by a higher dose of Nic (10 $\mathrm{mg} / \mathrm{kg} /$ day). Second, the ventricular dysfunction was not suppressed by these treatments in the acute phase, but the left ventricular function recovered better with treatment with Nic at 3 and $10 \mathrm{mg}$, or Mex at $25 \mathrm{mg}$ in the chronic phase. Third, the ventricular MAPD was prolonged in EAM in its acute phase as previously described, and this prolongation was suppressed by a higher dose of Mex ( $25 \mathrm{mg} / \mathrm{kg} /$ day $)$. Finally, although the level of the ATP and P-Cr was decreased in the myocardial mitochondria in the acute phase of the EAM regardless of the treatment, the redox state recovered better in the chronic phase with Nic treatment.

Cardioprotection by the use of K-ATP channel openers: The cardioprotective effect of Nic is known to be associated with a pharmacological preconditioning effect in acute ischemia. ${ }^{3,20,21)}$ Although two possible mechanisms for this cardioprotective effect have been discussed, a reduction in the intracellular calcium overload by shortening the action potential duration through sarcolemmal K-ATP channel opening and the protection of mitochondrial function by opening the mitochondrial $\mathrm{K}$-ATP channels, the latter has been more emphasized in recent reports because the effect of Nic on mitochondrial K-ATP channels is much stronger than that on sarcolemmal K-ATP channels. ${ }^{21-23)}$ Therefore, Nic is now mainly viewed as a mitochondrial K-ATP channel opener, at least at the clinical dose. In contrast, we previously documented the cardioprotective effect of Mex in an acute ischemia model. ${ }^{4}$ Although Mex has sodium channel blocking effects as well as sarcolemmal KATP channel opening effects, we concluded that the cardioprotective effect of Mex depended on the sarcolemmal K-ATP channel opening effect because its cardioprotective effect was totally suppressed by HMR-1098, the selective sarcolemmal K-ATP channel blocker used in the study. ${ }^{4}$

The time span of the myocardial injury in acute myocarditis is basically different from acute ischemia, in other words, the former appears in days or weeks, but the latter appears in minutes or hours. However, from the point of view of the process of the appearance of the final ventricular dysfunction, they are similar to each other because myocardial injury in the ear- lier phase results in ventricular dysfunction in the later phase in both diseases. In the present study, we hypothesized that myocardial protection utilizing K-ATP channel openers in the acute phase of myocarditis would lead to less ventricular dysfunction in the chronic phase similar to the acute ischemic model, and the result was that both Nic and Mex, mitochondrial and sarcolemmal K-ATP channel openers, exhibited a cardioprotective effect in the EAM model. To the best of our knowledge, this is the first documentation of the possibility of K-ATP channel openers being used for cardioprotection even in subacute or chronic ventricular dysfunction.

Mechanisms of the cardioprotection of K-ATP channel openers: The myocardial damage in EAM is considered to be caused by tissue inflammation including the myocardium and intercellular matrix. ${ }^{13,14)}$ It has been reported that acute inflammation causes myocardial damage, apoptosis, or necrosis and results in an intracellular and mitochondrial calcium overload similar to acute ischemia. ${ }^{7-10)}$ Therefore, a cardioprotective effect would be expected with a treatment which would reduce the calcium overload in the myocardium or its mitochondria. In the present study, we used two different clinically available KATP channel openers. As described above, Nic is mainly a mitochondrial K-ATP channel opener and Mex is a sarcolemmal K-ATP channel opener. As a result, Mex did not have an affect on the myocarditis itself, but a higher dose of Mex shortened the ventricular MAPD in the acute phase probably due to sarcolemmal K-ATP channel opening, and it led to better ventricular function in the chronic phase. This result indicates that shortening the myocardial action potential duration in the acute phase of myocardial injury may result in later myocardial protection possibly through the reduction in the intracellular calcium overload. ${ }^{4,20,21)}$ In contrast, Nic affected neither the myocarditis nor MAPD in the acute phase, but the Nic treatment resulted in better ventricular function in the chronic phase. Because the redox state of the mitochondria improved in the EAM+Nic groups in comparison with the control EAM group, protection of the mitochondrial function during the acute phase seemed to be the mechanism of the cardioprotection by Nic, similar to the acute ischemia model. ${ }^{3-6,22)}$

Clinical implications: The results of this study indicate the possibility of a reduction in myocardial or mitochondrial calcium overload as a cardioprotective therapy even in acute myocarditis as it is in acute ischemia. Therefore, this kind of therapeutic approach might result in better cardiac function in the later phase of clinical myocarditis. Additionally, because the action potential duration is reportedly prolonged in patients with heart failure of any cause, intracellular calcium overload may play a role in promoting chronic myocardial injury. Therefore, a similar therapeutic approach, that is, a reduction in the calcium overload, might be a supportive therapy to obtain better ventricular function in the later phase in patients with heart failure.

Limitations: There are several limitations to the present study. First, the influence of the additional effects of the drugs, ie, the sarcolemmal K-ATP channel opening effect of Nic and sodium channel blocking effect of Mex, were not separated. This point can be discussed more specifically when using more specific K-ATP channel openers, such as diazoxide (selective mitochondrial K-ATP channel opener) and P1075 (selective sarcolemmal K-ATP channel opener) but they are not available for continuous oral administration. Second, additional studies uti- 
lizing specific blockers such as 5-HD (5-hydroxydecanoate, mitochondrial K-ATP channel blocker) or HMR 1098 (sarcolemmal K-ATP channel blocker) were not performed. ${ }^{4}$ However, they too are not available for long-term continuous oral administration. Finally, the effects of the drugs on the inflammation itself were not evaluated.

Conclusions: The effects of two clinically available K-ATP channel openers, Nic and Mex, were evaluated in EAM rats. Although Nic and Mex treatments did not exhibit any beneficial effects in the acute phase, they resulted in better ventricular function in the chronic phase. Nic, a mitochondrial K-ATP channel opener, exhibited a cardioprotective effect in EAM possibly through the protection of the mitochondrial function. Mex, a sarcolemmal K-ATP channel opener, also exhibited a cardioprotective effect in EAM in the chronic phase and suppressed the MAPD prolongation in the acute phase. Treatments utilizing K-ATP channel openers might be useful as cardioprotective therapies even in chronic or subacute heart failure.

\section{REFERENCES}

1. Wilde AA, Janse MJ. Electrophysiological effects of ATP sensitive potassium channel modulation: implications for arrhythmogenesis. Cardiovasc Res 1994; 28: 16-24. (Review)

2. Melchert PJ, Duncker DJ, Traverse JH, Bache RJ. Role of K+ATP channels and adenosine in regulation of coronary blood flow in the hypertrophied left ventricle. Am J Physiol 1999; 277: H617-25.

3. Ito $\mathrm{H}$, Taniyama $\mathrm{Y}$, Iwakura $\mathrm{K}$, et al. Intravenous nicorandil can preserve microvascular integrity and myocardial viability in patients with reperfused anterior wall myocardial infarction. J Am Coll Cardiol 1999; 33: 654-60.

4. Niwano S, Inuo K, Morohoshi Y, et al. Mexiletine protects myocardium during acute ischemia by opening sarcolemmal K-ATP channel: studies in closed-chest acute ischemia model in rabbits. J Cardiovasc Pharmacol 2004; 44: 639-44.

5. Suzuki M, Sasaki N, Miki T, et al. Role of sarcolemmal K(ATP) channels in cardioprotection against ischemia/reperfusion injury in mice. J Clin Invest 2002; 109: 509-16.

6. Shigematsu S, Sato T, Abe T, Saikawa T, Sakata T, Arita M. Pharmacological evidence for the persistent activation of ATP-sensitive $\mathrm{K}+$ channels in early phase of reperfusion and its protective role against myocardial stunning. Circulation 1995; 92: 2266-75.

7. Watzinger N, Lund GK, Higgins CB, Chujo M, Saeed M. Noninvasive assessment of the effects of nicorandil on left ventricular volumes and function in reperfused myocardial infarction. Cardiovasc Res 2002; 54 : 77-84

8. Khaliulin I, Schwalb H, Wang P, et al. Preconditioning improves postischemic mitochondrial function and diminishes oxidation of mitochondrial proteins. Free Radic Biol Med 2004; 37: 1-9.

9. Rousou AJ, Ericsson M, Federman M, Levitsky S, McCully JD. Opening of mitochondrial KATP channels enhances cardioprotection through the modulation of mitochondrial matrix volume, calcium accumulation, and respiration. Am J Physiol Heart Circ Physiol 2004; 287: H1967-76.

10. Sato T, Sasaki N, Seharaseyon J, O’Rourke B, Marbán E. Selective pharmacological agents implicate mitochondrial but not sarcolemmal K(ATP) channels in ischemic cardioprotection. Circulation 2000; 101: 2418-23.

11. Akar FG, Rosenbaum DS. Transmural electrophysiological heterogeneities underlying arrhythmogenesis in heart failure. Circ Res 2003; 93: 638-45.

12. Yatani A, Kim SJ, Kudej RK, et al. Insights into cardioprotection obtained from study of cellular $\mathrm{Ca} 2+$ handling in myocardium of true hibernating mammals. Am J Physiol Heart Circ Physiol 2004; 286: H2219-28.

13. Wakisaka Y, Niwano S, Niwano H, et al. Structural and electrical ventricular remodeling in rat acute myocarditis and subsequent heart failure. Cardiovasc Res 2004; 63: 689-99.

14. Saito J, Niwano S, Niwano H, et al. Electrical remodeling of the ventricular myocardium in myocarditis: studies of rat experimental autoimmune myocarditis. Circ J 2002; 66: 97-103.

15. He ZS, Komori S, Tamura K, Hashimoto K. Inhibitory effect of moricizine on reperfusion induced tachyarrhythmias in rats --a comparison study with disopyramide and mexiletine. Jpn Circ J 1992; 56: 861-5

16. Saeed M, Watzinger N, Krombach GA, et al. Left ventricular remodeling after infarction: sequential MR imaging with oral nicorandil therapy in rat model. Radiology 2002; 224: 830-7.

17. Sanada S, Node K, Asanuma H, et al. Opening of the adenosine triphosphate-sensitive potassium channel attenuates cardiac remodeling induced by long-term inhibition of nitric oxide synthesis: role of 70-kDA S6 kinase and extracellular signal-regulated kinase. J Am Coll Cardiol 2002; 40: 991-7.

18. Masuda T, Dobson GP, Veech RL. The Gibbs-Donnan near-equilibrium system of heart. J Biol Chem 1990; 265: 20321-34

19. Sato K, Kashiwaya Y, Keon CA, et al. Insulin, ketone bodies, and mitochondrial energy transduction. FASEB J 1995; 9: 651-8.

20. Imagawa J, Baxter GF, Yellon DM. Myocardial protection afforded by nicorandil and ischaemic preconditioning in a rabbit infarct model in vivo. J Cardiovasc Pharmacol 1998; 31: 74-9.

21. Sato T, Sasaki N, O’Rourke B, Marbán E. Nicorandil, a potent cardioprotective agent, acts by opening mitochondrial ATP-dependent potassium channels. J Am Coll Cardiol 2000; 35: 514-8.

22. IONA Study Group. Effect of nicorandil on coronary events in patients with stable angina: the Impact Of Nicorandil in Angina (IONA) randomised trial. Lancet 2002; 359: 1269-75.

23. Murata M, Akao M, O’Rourke B, Marbán E. Mitochondrial ATPsensitive potassium channels attenuate matrix $\mathrm{Ca}(2+)$ overload during simulated ischemia and reperfusion: possible mechanism of cardioprotection. Circ Res 2001; 89: 891-8. 